\title{
Valoración ambiental de los ecosistemas leníticos del "Parque Regional en torno a los ejes de los cursos bajos de los ríos Manzanares y Jarama" (Madrid, España)
}

\author{
Neftalí Roblas \& Javier García-Avilés \\ Centro de Investigaciones Ambientales de la Comunidad de Madrid "Fernando González Bernáldez". C/ San \\ Sebastián 71, 28791 SOTO DEL REAL (Madrid), España.
}

\begin{abstract}
RESUMEN
La principal finalidad de este estudio consiste en establecer el valor ambiental de algunos ecosistemas leniticos localizados en una de las más importantes áreas protegidas de Madrid, el "Parque Regional en Torno a los Ejes de los Cursos Bajos de los Ríos Manzanares y Jarama". Para establecer el valor de cada ecosistema se desarrolló un índice semicuantitativo siguiendo los siguientes siete criterios: singularidad/vulnerabilidad de las especies de peces y aves presentes, riqueza especifica (basada en la presencia de peces y aves), representatividad del cuerpo de agua, grado de naturalidad, valor paisajístico/recreativo/educativo, viabilidad de su recuperación y grado de los impactos humanos. Los resultados muestran que entre los 123 ecosistemas acuáticos localizados en el Parque, 28 (23\%) presentan un elevado valor ambiental, 23 (19\%) un valor intermedio y 72 (58\%) valores bajos. Aqui se ha mostrado un profundo impacto humano en los ecosistemas lénticos de esta Área Natural Protegida. De hecho, las actividades humanas son consideradas el principal agente de formación (90\% de los ecosistemas leníticos del Parque son lagunas de gravera). Aún más, estas actividades mineras han generado todos los cuerpos de agua con elevado valor ambiental presentes en el área de estudio.
\end{abstract}

Palabras clave: Valoración ambiental, ecosistemas leniticos, gravera, extracción de áridos, Jarama, Manzanares, Madrid.

\begin{abstract}
The environmental value of lentic ecosystems in one of the most important protected areas in Madrid was established ("Parque Regional en torno a los ejes de los cursos bajos de los ríos Manzanares y Jarama"). This Park is a Nature Reserve located near Rivers Manzanares and Jarama. A semi-quantitative index was developed following seven criteria, i.e. singularity/vulnerability of fish and bird species present, species richness (fish and bird species), water body representativity, "naturalness", landscape/recreational/educational value, viability of recovery, and degree of human impacts. Results show that among the 123 lagoons in the Park, 28 (23\%) have high environmental value, $23(19 \%)$ an intermediate value, and $72(58 \%)$ are deemed to have little environmental value. A deep human impact on these lagoons, located within a Nature Reserve is apparent. Human activity is the main driver of ecological pattern formation in the Park. Indeed, $90 \%$ of lentic ecosystems in the Park are gravel-pit lakes. Moreover, mining activities and natural recovery have generated lagoons of high environmental value.
\end{abstract}

Keywords: Environmental value, lentic ecosystems, gravel-pit lake, sand extraction, Jarama, Manzanares, Madrid.

\section{INTRODUCCIÓN}

La valoración ambiental de ecosistemas constituye una herramienta enormemente útil de cara a su gestión, ya que así pueden definirse las directrices y prioridades de actuación para la protección de los mismos y la optimización de los usos que pueden albergar de acuerdo a sus características ecológicas. En el presente 
estudio se aborda la valoración de los ecosistemas acuáticos leníticos que se localizan en uno de los Espacios Naturales Protegidos de la Comunidad de Madrid: el "Parque Regional en torno a los ejes de los cursos bajos de los ríos Manzanares y Jarama”. Para ello se han analizado diversos aspectos ecológicos de estos medios.

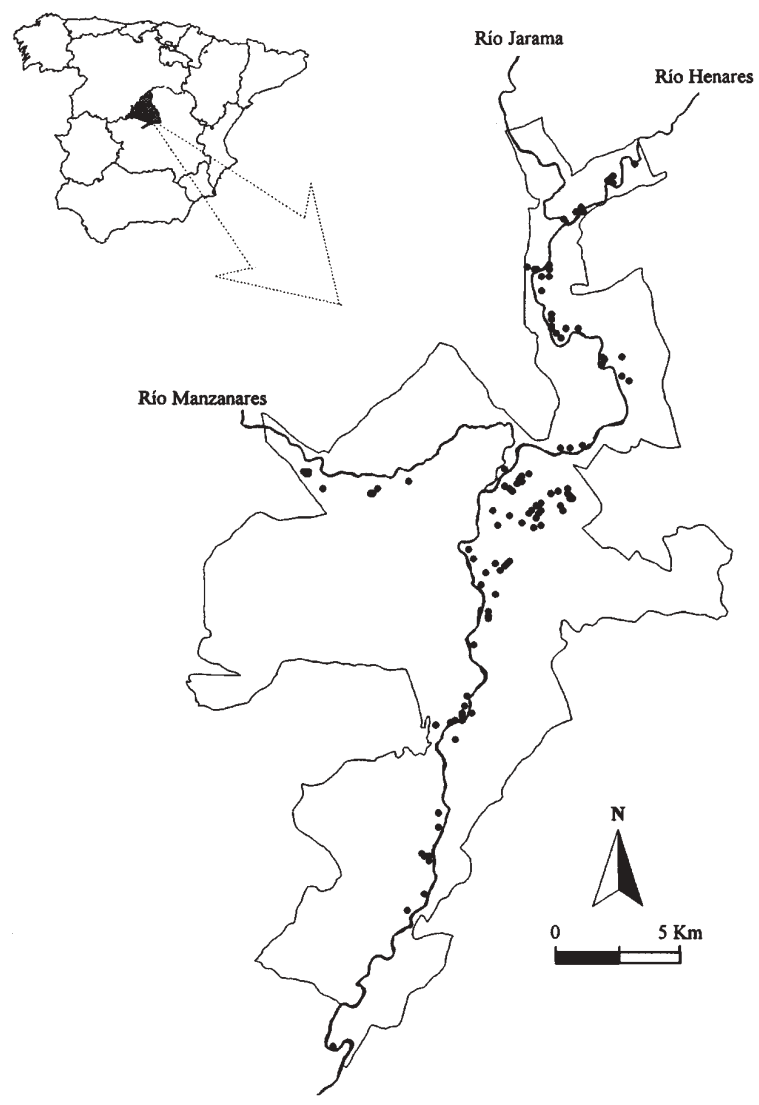

Figura 1. Mapa de localización del "Parque Regional en torno a los ejes de los cursos bajos de los ríos Manzanares y Jarama" y de los ecosistemas acuáticos leníticos. Situation map of the Park called "Parque Regional en torno a los ejes de los cursos bajos de los ríos Manzanares y Jarama" and location of the lagoons within the Park.

\section{ÁREA DE ESTUDIO}

El "Parque Regional en torno a los ejes de los cursos bajos de los ríos Manzanares y Jarama" fue creado en Junio de 1994 (Comunidad de Madrid, 1994). Su extensión es de aproximadamente $300 \mathrm{~km}^{2}$ y se encuentra ubicado en el Sureste de la Comunidad de Madrid (Fig. 1), ocupando una banda de territorio en sentido Norte-Sur que recorre los cursos fluviales del río Jarama y, en menor medida, del Manzanares y Henares.

Las vegas bajas del Manzanares y del Jarama son un ejemplo paradigmático de sustitución acelerada de usos del suelo (García et al., 1996), siendo varios los procesos que han definido el fuerte deterioro que ha venido sufriendo este territorio a partir de los años 60. Por un lado se ha producido una gran expansión de los núcleos de población, de forma simultánea al abandono de las tierras de cultivo que antaño dibujaron un paisaje de típica vega tradicional. Acompañando a estos fenómenos han proliferado las actividades industriales y extractivas que han sustituido los usos agrícolas tradicionales, destacando sobre todo la actividad minera de extracción de áridos que se ha venido desarrollando para el abastecimiento de materiales de construcción, como consecuencia de la expansión del área metropolitana de Madrid y su entorno.

La extracción de áridos de las Vegas Fluviales de los ríos que recorren el Parque (Henares, Jarama y Manzanares), ha generado depresiones que interceptan la superficie freática del acuífero Cuaternario Aluvial asociado a estas formaciones geológicas. Como consecuencia de ello, esta actividad minera altamente impactante ha contribuido a generar 111 ecosistemas acuáticos dentro del Parque; más del $90 \%$ de las láminas de agua que aparecen en él. El resto de ecosistemas acuáticos artificiales presentes en el área de estudio corresponden a 2 embalses, una balsa de riego, y un pozo. Tan solo 8 ecosistemas acuáticos de los 123 localizados en el Parque (el 6,5 \%) tienen un origen natural. Se trata de siete charcas asociadas a manantiales y de una laguna marginal. 
La superficie total ocupada por láminas de agua dentro del Parque es de 411,1 ha. El tamaño de los ecosistemas estudiados resulta altamente heterogéneo, apareciendo desde humedales que ocupan escasos metros cuadrados, como es el caso de las charcas originadas por manantiales, hasta algunas lagunas de gravera que alcanzan superficies de varias decenas de hectáreas

\section{METODOLOGÍA}

Existe una gran variedad de criterios a la hora de realizar la valoración ambiental de los ecosistemas presentes en un territorio (Amat et al., 1985; Cirujano et al., 1992; Montes, 1993; Eurosite, 1996; Cadiñanos, 1997; Usher, 1997). Los más utilizados tradicionalmente han sido criterios tales como la riqueza de especies, la rareza o singularidad de éstas, la representatividad de los ecosistemas, el grado de interferencia humana, los valores educativos, los usos potenciales, etc.

De todos ellos, para la valoración de los ecosistemas leníticos del Parque se han utilizado siete criterios que definen las propiedades de cada medio, tanto a nivel ecológico como en función de la potencialidad de cara a su aprovechamiento y gestión (Tabla 1). Estos criterios pueden agruparse en tres clases: biológicos, ecológicos y antrópicos.

Los criterios biológicos utilizados han sido la singularidad o vulnerabilidad de especies presentes en los ecosistemas y la riqueza especifica. Para ello, en ambos casos, se han considerando los grupos faunísticos presentes en estos medios sobre los que se posee más información; peces y aves (si bien en el caso de las aves solo se posee información por complejos lagunares dada la alta movilidad de estos organismos). En el caso de la singularidad/vulnerabilidad de las especies, se ha

Tabla 1. Criterios utilizados en la valoración ambiental de los ecosistemas leníticos del Parque y rangos asignados a cada variable. Criteria used in the environmental assessment of lentic ecosystems of the Nature Reserve ("Parque Regional en torno a los ejes de los cursos bajos de los rios Manzanares y Jarama"; Madrid, Spain) and ranks assigned to each variable.

\begin{tabular}{|c|c|c|c|c|}
\hline \multirow[t]{2}{*}{ CRITERIOS } & \multicolumn{4}{|c|}{ RANGOS } \\
\hline & 0 & 1 & 2 & 3 \\
\hline \multicolumn{5}{|l|}{ Biológicos } \\
\hline $\begin{array}{l}\text { Singularidad/ } \\
\text { vulnerabilidad } \\
\text { de especies }\end{array}$ & $\begin{array}{l}\text { Sin especies } \\
\text { catalogadas }(1,2,3)\end{array}$ & "Especial interés" (1) & $\begin{array}{l}\text { "Vulnerables" }{ }^{(1)}, \\
\text { "Especial interés }{ }^{(2)}, \\
\text { "Raras"(3) }\end{array}$ & $\begin{array}{l}\text { Máxima vulnerabilidad/ } \\
\text { singularidad }^{(1,2,3)}\end{array}$ \\
\hline Riqueza de especies (\%) & $0-5 \%$ & $6-15 \%$ & $16-50 \%$ & $>50 \%$ \\
\hline \multicolumn{5}{|l|}{ Ecológicos } \\
\hline Representatividad & Nula & Baja & Media & Alta \\
\hline Grado de naturalidad & Muy bajo & Bajo & Medio & Alto \\
\hline \multicolumn{5}{|l|}{ Antrópicos } \\
\hline $\begin{array}{l}\text { Valor paisajístico, } \\
\text { recreativo y educativo }\end{array}$ & Nulo & Bajo & Medio & Alto \\
\hline Viabilidad de recuperación & Nula & Baja & Media & Alta \\
\hline $\begin{array}{l}\text { Porcentaje de impactos } \\
\text { e intensidad }\end{array}$ & $>30 \% /$ Alta & $15-30 \% /$ Media & $5-15 \% /$ Baja & $<5 \% /$ Muy baja \\
\hline
\end{tabular}

(1): Comunidad de Madrid, 1992. (2): MAPA, 1990. (3): Blanco \& González, 1992. 
tenido en cuenta cuales de ellas están catalogadas con alguna figura de protección o grado de amenaza (M.A.P.A., 1990; Blanco \& González, 1992; Comunidad de Madrid, 1992). En cuanto a la riqueza de específica de los humedales estudiados, se ha considerado el número de especies presentes en cada uno de ellos en relación al número de especies presentes en el conjunto de ecosistemas leníticos del Parque (Tabla 1).

En cuanto al segundo grupo de criterios, los ecológicos, hacen referencia por un lado a la representatividad de los humedales en base a una tipificación previamente realizada de estos medios (Roblas \& García-Aviles, 1999). En ella se clasifica al total de las láminas de agua generadas por actividades extractivas del Parque en ocho grupos de características ambientales homogéneas. Para ello, son utilizadas las ocho variables que mejor caracterizan a los ecosistemas estudiados: litología sobre la que se genera la cubeta, distancia al cauce fluvial, superficie de la lámina de agua, sinuosidad de la línea de costa, grado de encajamiento de la cubeta, granulometría del sustrato de la cubeta, vegetación del entorno, y el grado de desarrollo de la vegetación helófita del propio humedal.

Por otra parte, teniendo en cuenta que el 93,5 $\%$ de los humedales del Parque son artificiales, se ha considerado el grado de naturalidad de éstos, evaluado en base al desarrollo de la vegetación helófita. Esta variable constituye un reflejo de múltiples factores ecológicos que definen la organización y funcionamiento de los humedales (encajamiento de la cubeta, granulometría del sustrato, antigüedad de la lámina de agua y tiempo de abandono, usos e impactos a los que están sometidos, trabajos de restauración que ha experimentado el humedal, etc.), condensando una gran cantidad de información en un solo parámetro fácilmente medible.

Por último, debido al origen antrópico de la mayor parte de los ecosistemas estudiados y a la fuerte influencia que la actividad humana tiene sobre muchos de ellos, se han incluido tres criterios que pretenden reflejar la repercusión sobre el valor ambiental de cada humedal, tanto de los impactos que se ejercen sobre ellos, como de los usos actuales y potenciales que presentan. El primero de estos criterios es el valor paisajístico, recreativo y educativo de cada humedal, en función de si han sido sometidos a restauración, albergan programas de educación ambiental, o han sido acondicionados para su uso recreativo, por parte de la administración local o autonómica. En segundo lugar, se ha considerado la viabilidad de recuperación de los ecosistemas por su potencialidad para ser destinado a los usos anteriores en función de la proximidad a núcleos de población, accesibilidad, estado de conservación, etc. Por último, se han tenido en cuenta los impactos a los que se ven sometidos los ecosistemas leníticos del Parque, considerando tanto el porcentaje de éstos que sufre cada lámina de agua con respecto al total de los impactos detectados en el conjunto de humedales del Parque, como la intensidad de los mismos.

\section{RESULTADOS}

A partir de los siete criterios expuestos anteriormente se ha generado un índice de valoración $\left(\mathrm{I}^{\mathrm{v}}\right)$, resultado de la suma de cada uno de los valores asignados para cada criterio a cada humedal. Dentro de este gradiente continuo de valoración se han establecido tres clases:

- Valoración ambiental alta ( $\left.\mathrm{I}^{\mathrm{V}} \geq 18\right)$ : Humedales que presentan valores biológicos relevantes (especies raras o amenazadas, comunidades bien desarrolladas, etc.), así como otras características ecológicas, paisajísticas, pedagógicas, etc., que les confieren importancia a nivel internacional, nacional o regional. La inclusión de un humedal en esta categoría le asigna un carácter preferente para su conservación.

- Valoración ambiental media $\left(18>\mathrm{I}^{\mathrm{v}}=12\right)$ : Humedales que sin presentar las características relevantes de la clase anterior, poseen valores ecológicos e importancia paisajística, singularidad, etc., o representan un marco adecuado para las diversas activida- 
Tabla 2. Valoración ambiental de los ecosistemas leníticos del "Parque Regional en torno a los ejes de los cursos bajos de los ríos Manzanares y Jarama". Environmental value of lentic ecosystems of the Nature Reserve called "Parque Regional en torno a los ejes de los cursos bajos de los rios Manzanares y Jarama” (Madrid, Spain).

\begin{tabular}{|c|c|c|c|}
\hline \multicolumn{2}{|c|}{ Valoración } & \multicolumn{2}{|l|}{ Nombre } \\
\hline \multirow[t]{10}{*}{ ALTA } & Embalse de Gózquez & Lagunas del Campillo 2 & Graveras Pioneer 6 \\
\hline & Graveras del Río Henares 2 & Lagunas del Campillo 3 & Graveras Pioneer 7 \\
\hline & Graveras del Río Henares 3 & Graveras de San Martín de la Vega 3 & Gravera el Picón \\
\hline & Graveras del Río Henares 4 & Graveras de San Martín de la Vega 4 & Lagunas El Porcal 4 \\
\hline & Graveras del Río Henares 5 & Graveras de San Martín de la Vega 5 & Lagunas El Porcal 5 \\
\hline & Graveras del Río Henares 6 & Lagunas de Las Madres 4 & Lagunas El Porcal 6 \\
\hline & Lagunas de la Presa del Río Henares 1 & Lagunas de Velilla 3 & Lagunas El Porcal 7 \\
\hline & Lagunas de la Presa del Río Henares 2 & Gravera Soto de las Cuevas & Lagunas de Ciempozuelos 1 \\
\hline & Lagunas de la Presa del Río Henares 3 & Graveras Pioneer 5 & Lagunas de Ciempozuelos 2 \\
\hline & Lagunas de la Presa del Río Henares 4 & & \\
\hline \multirow[t]{8}{*}{ MEDIA } & Graveras del Río Henares 1 & Graveras Pioneer 4 & Lagunas de Velilla 1 \\
\hline & Lagunas de la Presa del Río Henares 5 & Lagunas de Velilla 2 & Lagunas de Las Madres 2 \\
\hline & Lagunas del Campillo 1 & Lagunas El Porcal 8 & Manantial La Boyeriza \\
\hline & Graveras de San Martín de la Vega 1 & Lagunas El Porcal 9 & Salinas de Espartinas \\
\hline & Graveras de San Martín de la Vega 2 & Lagunas de Las Madres 1 & Manantial Casa Eulogio \\
\hline & Graveras Pioneer 1 & Lagunas de Las Madres 3 & Manantial Casa Soto de Rivas \\
\hline & Graveras Pioneer 2 & Graveras Soto Pajares 3 & Manantial Los Albergues \\
\hline & Graveras Pioneer 3 & Graveras Soto Pajares 4 & \\
\hline \multirow[t]{24}{*}{ BAJA } & Balsa de Riego Casas de San Medino & Gravera Los Angeles 2 & Graveras Soto Pajares 2 \\
\hline & Presa del Rey & Gravera Soto Gutierrez 2 & Graveras Soto Pajares 6 \\
\hline & Gravera El Negralejo & Graveras de Rivas 1 & Gravera Maresa 1 \\
\hline & Graveras de Rivas 2 & Graveras de San Antonio 3 & Gravera Casa de Castro 2 \\
\hline & Graveras de Rivas 3 & Graveras de San Antonio 4 & Gravera El Charcón 5 \\
\hline & Graveras Corviam 1 & Graveras de San Antonio 5 & Lagunas El Porcal 2 \\
\hline & Graveras Corviam 2 & Gravera Las Mantecas 1 & Graveras Soto Pajares 1 \\
\hline & Graveras Corviam 3 & Graveras Camping Lagos 1 & Graveras Soto Pajares 7 \\
\hline & Graveras Soto Pajares 5 & Graveras Camping Lagos 2 & Gravera Maresa 2 \\
\hline & Gravera Isla del Herrero & Laguna Muñoz & Gravera Soto de la Chopera 1 \\
\hline & Graveras de San Martín de la Vega 6 & Lagunas de Evencio Niño 1 & Gravera Soto de la Chopera 2 \\
\hline & Graveras de San Martín de la Vega 7 & Lagunas de Evencio Niño 2 & Gravera Soto de la Chopera 4 \\
\hline & Gravera Ticosa 1 & Laguna Villafranca & Gravera Casa de Castro 1 \\
\hline & Gravera Ticosa 2 & Laguna Ulibarri & Gravera El Charcón 1 \\
\hline & Graveras de Rivas 4 & Graveras La Esperilla 1 & Gravera El Charcón 2 \\
\hline & Graveras de Rivas 5 & Graveras La Esperilla 2 & Gravera El Charcón 3 \\
\hline & Graveras de Arganda 1 & Graveras La Conejera 4 & Gravera El Charcón 4 \\
\hline & Graveras de Arganda 2 & Graveras La Conejera 1 & Gravera Maresa 3 \\
\hline & Gravera Los Angeles 3 & Graveras La Conejera 2 & Gravera Soto Gutierrez 1 \\
\hline & Gravera Los Angeles 1 & Gravera Parque Tierno Galván & Gravera Soto de la Chopera 3 \\
\hline & Gravera Casas de San Medino & Lagunas El Porcal 1 & Laguna marginal del Jarama \\
\hline & Gravera Casa de Castro 3 & Lagunas El Porcal 3 & Fuente de Palomero \\
\hline & Laguna de los Frailes & Lagunas El Porcal 10 & Manantial Casa del Congosto \\
\hline & Gravera Soto las Juntas & Gravera Las Mantecas 2 & Manantial Calamuecos \\
\hline
\end{tabular}


des recreativas respetuosas con la naturaleza, así como valores pedagógicos; siendo enclaves a mejorar y conservar dentro del marco del Parque Regional, ante la viabilidad de su uso racional.

- Valoración ambiental baja $\left(\mathrm{I}^{\mathrm{V}}<12\right)$ : Humedales sin ningún tipo de valor ambiental a destacar, generalmente altamente degradados, sometidos a fuertes impactos o de muy reciente creación. Se trata fundamentalmente de láminas de agua generadas por actividades extractivas en zonas de explotación o utilizadas como vertederos.

Como resultado de la valoración ambiental de los 123 ecosistemas acuáticos leníticos presentes en el Parque Regional, el 23 \% (28 humedales) han obtenido una valoración ambiental alta, el $19 \%$ (23 casos) una valoración media y el $58 \%$ restante (72 de los ecosistemas estudiados) aparecen dentro de la clase de valoración baja (Tabla 2).

\section{DISCUSIÓN}

La valoración ambiental de unos ecosistemas acuáticos que en su mayoría tienen un origen artificial es, cuando menos, una tarea compleja y nunca definitiva. Está proceso resulta siempre en mayor o menor medida subjetivo, pero constituye una herramienta muy útil a la hora de definir prioridades de conservación y políticas enfocadas a la gestión racional de los ecosistemas. En este sentido, los criterios utilizados podrían considerarse subjetivos, pero sin embargo, caracterizan y diferencian claramente a las láminas de agua del Parque. Por ello, proporcionan una herramienta enormemente valiosa que nos permite, no asignar un valor absoluto a cada humedal, sino contrastar y relativizar el valor ambiental y estado de conservación de unos ecosistemas en relación a otros, en base a los criterios previamente fijados. Esto a su vez nos permitirá definir políticas de actuación una vez evaluada la situación de los humedales del Parque. Además, el método de valoración ambiental expuesto nos proporciona una forma de analizar la evolución del estado de conservación de los humedales del Parque a través de estudios futuros en los que se aplique la misma metodología, así como su comparación con otros ecosistemas.

De los 28 humedales que reciben una valoración ambiental alta, 27 se corresponden con lagunas de gravera que forman complejos lagunares de gran importancia en una región como es la Comunidad de Madrid, que presenta un bajo número de zonas húmedas de origen natural, hecho que ha motivado en parte que estos ecosistemas artificiales se encuentren incluidos en el "Catálogo de Embalses y Humedales de la Comunidad de Madrid" para su protección (Comunidad de Madrid, 1991). Se trata de láminas de agua que en muchos casos han sido sometidas a planes de restauración destinados a potenciar sus valores para albergar usos recreativos, educativos o como "reservas ecológicas". Asimismo destacan por albergar importantes comunidades orníticas que les confieren relevancia a nivel regional o nacional. El otro medio lenítico incluido en esta clase es el embalse de Gózquez, que tiene su origen en el represamiento de aguas para el riego de cultivos en la actualidad abandonados, testimonio de los usos agrícolas que tradicionalmente se llevaban a cabo en la zona. Este presenta una elevada riqueza de aves, además de un gran valor paisajístico.

Dentro de los ecosistemas leníticos que alcanzan una valoración ambiental media, existen dos grupos que merecen ser destacados. Por un lado, están aquellas lagunas de gravera que forman parte de complejos lagunares en los que sí hay otros humedales con valoración ambiental alta. Son sobre todo estas lagunas las que resultan prioritarias a la hora de ser recuperadas, ya que a través de las medidas de restauración y gestión apropiadas en cada caso pueden ver aumentados sus valores ambientales significativamente, uniéndose a aquellas que estando muy próximas dentro de los complejos, alcanzan la máxima valoración. 
El otro grupo a mencionar, dentro de la valoración media, lo componen los medios acuáticos de origen natural, fundamentalmente manantiales, que han quedado infravalorados en el presente estudio ya que no poseen comunidades de vertebrados relevantes y que debido a su pequeño tamaño no presentan una clara potencialidad de usos; siendo sin embargo unos hábitats de gran interés limnológico enclavados en un territorio de características semiáridas.

Hay que destacar el dominio de los humedales con valoración ambiental baja (58\%), lo que está en consonancia con el carácter artificial de la mayoría de los ecosistemas estudiados y con la fuerte influencia antrópica que presenta todo el Parque Regional, lo que hace muy difícil los procesos de naturalización. Estos medios, altamente degradados, son en su mayoría lagunas de gravera que están ubicadas bien en zonas de explotación activa o que, por el contrario, han sido abandonadas completamente, sufriendo numerosos e intensos impactos.

Finalmente es necesario resaltar la paradoja que supone que una actividad altamente impactante como es la extracción de áridos, haya dado lugar al mismo tiempo a una fuerte degradación del entorno y a la creación de unos ecosistemas nuevos, las lagunas de gravera, que debido a su gran riqueza ornitológica han supuesto uno de los motivos fundamentales por los que fue declarado el Parque. Este hecho refleja la necesidad de una visión integral del territorio para su gestión y conservación, y no atendiendo a elementos particulares dentro de los ecosistemas. La consecuencia directa de la confrontación entre la conservación y la explotación intensiva del territorio, es la fuerte polémica a la que está sometido este espacio natural protegido desde su creación.

\section{BIBLIOGRAFÍA}

AMAT, J. A., C. DÍAZ PANIAGUA, C. M. HERRERA, P. JORDANO, J. R. OBESO \& R. C. SORIGUER. 1985. Criterios de valoración de zonas húmedas de importancia nacional y regional en función de las aves acuáticas. M.A.P.A. Monografias $35.79 \mathrm{pp}$.

BLANCO, J. C. \& J. L. GONZÁLEZ (eds.), 1992. Libro rojo de los vertebrados de España. ICONA, M.A.P.A. 714 pp.

CADIÑANOS, J. A. 1997. Valoración del interés naturalístico y de conservación de unidades de vegetación: Problemática, propuestas metodológicas y aplicación en la Reserva de la Biosfera de Urdaibai (Vizcaya). Tesis Doctoral, Univ. del País Vasco. 341 pp.

CIRUJANO, S., M. VELAYOS, F. CASTILLA \& M. GIL. 1992. Criterios botánicos para la valoración de las lagunas y humedales españoles (Península Ibérica y las Islas Baleares). ICONA-C.S.I.C. 456 pp.

COMUNIDAD DE MADRID. 1991. Acuerdo de 10 de octubre de 1991, del Consejo de Gobierno, por el que se aprueba el Catálogo de Embalses y Humedales de la Comunidad de Madrid. B.O.C.M., 257: 4-6.

COMUNIDAD DE MADRID. 1992. Decreto 18/92, de 26 de marzo, por el que se aprueba el Catálogo Regional de especies amenazadas de fauna y flora silvestres y se crea la categoría de árboles singulares. B.O.C.M., 85: 5-11.

COMUNIDAD DE MADRID. 1994. Ley 6/1994, de 28 de junio, sobre el Parque Regional en torno a los ejes de los cursos bajos de los ríos Manzanares y Jarama. B.O.C.M., 163: 2-22.

GARCÍA, R., I. GONZÁLEZ, A. ORDEJÓN \& C. ALFONSO. 1996. Evolución del paisaje rural en la vega baja del Jarama (Madrid). Actas de las VIII Jornadas Sobre el Paisaje. La conservación del paisaje rural, Asociación para el Estudio del Paisaje: 173-183.

EUROSITE, 1996. European Guide for the preparation of management plans for protected and managed natural and semi-natural areas. Management Plans: Methods and Techniques. EUROSITE. 50 pp.

M.A.P.A., 1990. Real Decreto 439/1990, de 30 de marzo, por el que se regula el Catálogo Nacional de Especies Amenazadas. B.O.E, 82: 9468-9471.

MONTES, C. (dir.), 1993. Estudio de los valores ambientales existentes en las láminas de agua generadas por actividades extractivas en la Comunidad de Madrid. Agencia de Medio Ambiente, Comunidad de Madrid; Departamento Interuniversitario de Ecología, Univ. Autónoma de Madrid. 234 pp. 
ROBLAS, N. \& J. GARCÍA-AVILÉS, 1999. Tipificación de las láminas de agua generadas por actividades extractivas del "Parque Regional en torno a los ejes de los cursos bajos de los ríos Manzanares y Jarama”. Limnetica, 17 :27-36.
USHER, M. B. 1997. Principles of nature conservation evaluation. In: Freshwater quality defining the indefinable. P.J. Boon \& D. L. Howell (eds.): 199214. Edinburgh Stationery Office. 